\title{
Efeito da pandemia pela COVID-19 no serviço de cirurgia e traumatologia
}

\section{bucomaxilofacial: um estudo comparativo}

\author{
Effect of COVID-19 pandemic in Oral and Maxillofacial Surgery and Traumatology: the \\ comparative study \\ Efecto de la pandemia COVID-19 en el servicio de cirugía y traumatología maxilofacial: un estudio \\ comparative
}

Recebido: 13/08/2021 | Revisado: 23/08/2021 | Aceito: 27/08/2021 | Publicado: 29/08/2021

Gabriela Bohneberger
ORCID: https://orcid.org/0000-0001-7025-9833
Universidade do Oeste do Paraná, Brasil
E-mail: gabibohne @ hotmail.com
Eleonor Álvaro Garbin Júnior
ORCID: https://orcid.org/0000-0002-2111-4766
Universidade do Oeste do Paraná, Brasil
E-mail: alvarogarbin @ yahoo.com.br
Geraldo Luiz Griza
ORCID: https://orcid.org/0000-0001-7169-495X
Universidade do Oeste do Paraná, Brasil
E-mail: ggriza @ hotmail.com
Evelyn Estefani Cristaldo Acosta
ORCID: https://orcid.org/0000-0003-4458-3888
Universidade do Oeste do Paraná, Brasil
E-mail: evelyn-acosta @ hotmail.com
Leticia Thais Otaviano
ORCID: https://orcid.org/0000-0002-7624-0348
Universidade do Oeste do Paraná, Brasil
E-mail: otavianoleticia5 @gmail.com
Natasha Magro Ernica
ORCID: https://orcid.org/0000-0002-0545-1623
Universidade do Oeste do Paraná, Brasil
E-mail: natashamagro@ uol.com.br

\section{Resumo}

A pandemia pela COVID-19 promoveu o comprometimento dos sistemas de saúde de diversos países. Este estudo retrospectivo foi realizado para avaliar o efeito provocado pela pandemia no serviço de Cirurgia e Traumatologia Bucomaxilofacial do Hospital Universitário do Oeste do Paraná, em Cascavel. Foram comparados os pacientes atendidos no período de pandemia, entre 16 de março de 2020 até 15 de março de 2021, com o mesmo período, um ano anterior, denominado pré-pandemia. Os dados foram categorizados com base no total de atendimentos, horário e data de admissão e etiologias. Variações de idade e sexo também foram avaliadas. Estatísticas foram admitidas com significância em $\mathrm{p}<0,05$. Os resultados demonstram que houve redução dos casos durante a pandemia $(\mathrm{n}=321)$ comparado com o período pré-pandemia $(n=423 \mathrm{p}=0,03)$. Também houve diminuição nas admissões noturnas entre os períodos $(n=126$ e $n=181$, respectivamente) com significância $(p=0,037)$. Em relação ao sexo, o perfil de pacientes masculinos apresentou redução $(n=220$ e $n=303, p=0,005)$. A etiologia de casos de agressão física apresentou redução significativa $(n=38$ e $n=64, p=0,016)$ assim como os casos de multifatorial $(n=49$ e $n=97, p=0,011)$. O período de pandemia mostrou associação com a redução de pacientes com trauma bucomaxilofacial. Concluiu-se que a redução de casos admitidos no período noturno assim como de pacientes do sexo masculino pode ser atribuída como resultado das restrições de circulação que a pandemia impôs. O efeito do consequente distanciamento social, sugere uma diminuição no trauma bucomaxilofacial devido à agressão física.

Palavras-chave: Bucomaxilofacial; Trauma; COVID-19.

\section{Abstract}

The COVID-19 pandemic promoted the commitment of the health system in many countries. This study retrospective was realized to evaluate the effect promoted for the pandemic in Oral and Maxillofacial Surgery and Traumatology Service at Hospital Universitario do Oeste do Paraná, in Cascavel. A sample with patients in the pandemic period, between March 16th, 2020, and March 15th, 2021, as compared with patients in the previous year, called pre- 
pandemic. The data was categorized by the total of patients, admission date and hourly, and etiology. Age variations and gender were evaluated also. Statistics were admitted with significance at $p<0.05$. The results show that there was a reduction in cases during the pandemic $(n=321)$ compared to the pre-pandemic period $(n=423 p=0.03)$. There was also a decrease in nighttime admissions between periods ( $n=126$ and $n=181$, respectively) with significance $(\mathrm{p}=0.037)$. Regarding gender, the profile of male patients showed a reduction $(\mathrm{n}=220$ and $\mathrm{n}=303$, $\mathrm{p}=0.005)$. The etiology of cases of physical aggression showed a significant reduction $(n=38$ and $n=64, p=0.016)$ as well as the cases of multifactorial $(n=49$ and $n=97, p=0.011)$. The period in pandemic shows association with the reduction of the patients with oral-maxillofacial trauma. The reduction of the cases in nighttime admitted as the male patient was being shown who resulted from the restriction of circulation that the pandemic imposed. The effect of consequent social distance showed a decrease in maxillofacial trauma due to physical aggression.

Keywords: Maxillofacial; Trauma; COVID-19.

\section{Resumen}

La pandemia de COVID-19 promovió el compromiso de los sistemas de salud en varios países. Este estudio retrospectivo se realizó para evaluar el efecto causado por la pandemia en el servicio de Cirugía y Traumatología Maxilofacial del Hospital Universitario de Oeste do Paraná, en Cascavel. Los pacientes tratados durante el período pandémico, entre el 16 de marzo de 2020 y el 15 de marzo de 2021, se compararon con el mismo período, un año antes, llamado prepandémico. Los datos se categorizaron según el número total de visitas, la hora y fecha de ingreso y las etiologías. También se evaluaron las variaciones de edad y sexo. Se admitieron estadísticas con significación de $\mathrm{p}$ $<0,05$. Los resultados demuestran que hubo una reducción de casos durante la pandemia $(n=321)$ en comparación con el período prepandémico $(\mathrm{n}=423 \mathrm{p}=0,03)$. También hubo una disminución en los ingresos nocturnos entre períodos $(\mathrm{n}=126$ y $\mathrm{n}=181$, respectivamente) con significación $(\mathrm{p}=0,037)$. En cuanto al sexo, el perfil de los pacientes varones mostró una reducción $(n=220$ y $n=303, p=0,005)$. La etiología de los casos de agresión física mostró una reducción significativa $(n=38$ y $n=64, p=0,016)$ así como los casos multifactoriales $(n=49$ y $n=97, p=0,011)$. El período pandémico se asoció con una reducción de pacientes con trauma maxilofacial. Se concluyó que la reducción de los casos ingresados por la noche así como en los pacientes masculinos se puede atribuir a las restricciones circulatorias impuestas por la pandemia. El efecto del distanciamiento social sugiere una disminución del trauma maxilofacial por agresión física.

Palabras clave: Maxilofacial; Trauma; COVID-19.

\section{Introdução}

Desde dezembro de 2019, quando foi registrado o primeiro caso de COVID-19 com internamento na China (Wu et al., 2020), os serviços de saúde pelo mundo precisaram de uma readequação. O vírus SARS-CoV-2 se propaga de forma rápida e provoca complicações respiratórias, dentre elas a pneumonia relacionada a COVID-19, o que causou aumento na demanda por cuidados de saúde específicos e promoveu o comprometimento dos tratamentos de diversas áreas no sistema de saúde do mundo todo (Onder, Rezza, \& Brusaferro, 2020; Zimmermann \& Nkenke, 2020).

Devido ao fato de que pacientes positivos para SARS-CoV-2 podem ser assintomáticos, todos os pacientes devem ser considerados infectados (Rothe et al., 2020). Os procedimentos que geram aerossóis requerem maior cuidado pelo aumento na transmissão aérea do vírus (World Health Organization, 2020a; Yang et al., 2020). Estes procedimentos incluem traqueotomia, aspiração das vias aéreas, drenagem de abscesso, irrigação de feridas, uso de dispositivos ultrassônicos ou piezoelétricos, uso de peças de mão de alta velocidade, entre outros (Zimmermann \& Nkenke, 2020). Por isso, todo paciente deve ser tratado como potencialmente infectado e algumas medidas de proteção tomadas como a redução no número de membros da equipe na sala de cirurgia; ventilação adequada nos ambientes; proteção para os olhos, como os óculos de proteção, luvas e máscaras com alto nível de proteção (FFP2 / N99 / N95) (World Health Organization, 2020b).

Há um risco potencial de escassez de recursos relacionados aos atendimentos, desde equipamentos de proteção individual até medicamentos (World Health Organization, 2020b). A escassez destes recursos pode exigir adiamento de cirurgia eletiva, a fim de reduzir a pressão sobre o sistema de saúde, devido a isso é muito importante ter um conceito claro em relação aos procedimentos prioritários relacionados à cirurgia e traumatologia bucomaxilofacial (Zimmermann \& Nkenke, 2020).

O objetivo deste estudo foi analisar comparativamente os dados demográficos e etiológicos dos pacientes acometidos 
por trauma ou complicações bucomaxilofaciais atendidos no Hospital Universitário do Oeste do Paraná, entre os períodos prépandemia COVID-19 e durante a pandemia, identificando as mudanças na quantidade, no perfil demográfico e na etiologia.

\section{Metodologia}

Para atender ao objetivo da pesquisa, foi planejado e implementado um estudo comparativo retrospectivo.

A amostra do estudo foi composta por pacientes que se apresentaram ao Hospital Universitário do Oeste do Paraná, em Cascavel, Brasil e que evoluíram com traumatismo ou complicações bucomaxilofaciais. A data inicial para o grupo durante a pandemia, foi definida de acordo com o Decreto n 4230 do estado do Paraná, como dia 16 de março de 2020 (Governo do Estado do Paraná, 2020). Foram incluídos na pesquisa os pacientes que se apresentaram para avaliação e tratamento de trauma e complicações bucomaxilofaciais durante o período de pandemia pela COVID-19 de 2020 e o mesmo período durante 2019 e que possuíam registros suficientes e adequados para abstração de dados. O critério de exclusão foram os pacientes com registros médicos inadequados que impediram a abstração de dados, tal como impossibilidade de identificação.

Foi aprovado pelo parecer 4.839.886 do Comitê de Ética e Pesquisa da Universidade do Oeste do Paraná.

A variável inicial foi a comparação da quantidade de pacientes atendidos entre os dois períodos do estudo. O grupo denominado como pré-pandemia incluiu aqueles que se apresentaram entre 16 de março de 2019 até 15 de março de 2020. O grupo de comparação durante a pandemia, incluiu aqueles que se apresentaram durante o mesmo período durante a pandemia da COVID-19 começando em 16 de março de 2020 até 15 de março de 2021.

Além da variável da quantidade de pacientes de trauma e complicações bucomaxilofaciais, foram incluídas as variáveis de apresentação inicial, demográficas e de etiologia. A variável de apresentação inicial, descrimina em qual turno o paciente foi admitido ao hospital, sendo, i) manhã: 6:00 até 11:59; ii) tarde: 12:00 até 17:59 e; iii) noite: 18:00 até 05:59. Dentre as variáveis demográficas, a variável sexo foi dividida em masculino e feminino. A variável idade, foi atribuído valor nominal da idade no momento do atendimento. Por fim, as variáveis de etiologia, foram classificadas como: acidente de trânsito, que incluíram acidentes automobilísticos, motociclístico e atropelamento; acidentes esportivos, quedas, como queda de nível ou queda de própria altura; acidente de trabalho; agressão física incluindo agressão direta, com arma branca ou arma de fogo; multifatorial que incluíam infecções e cirurgias eletivas; e outros.

A coleta de dados foi realizada usando dois métodos: 1) coleta das variáveis do estudo do registro de trauma institucional e; 2) verificação e confirmação das variáveis no sistema do HUOP.

Todos os dados foram tabulados e mantidos em uma planilha segura no Excel. As estatísticas descritivas foram calculadas para todas as variáveis do estudo em cada período. O Teste $\mathrm{T}$ para duas amostras foi utilizado para comparar a mudança entre cada item entre as duas amostras (Lunardi, 2020), ajustando para a significância de $\mathrm{P}<0.05$. Todas as análises estatísticas foram realizadas usando o Software Stata/SE 16.1.

\section{Resultados}

Ao todo, foram registrados 749 pacientes, sendo que destes, cinco foram excluídos por não haver dados registrados de forma suficiente. Dos 744 restantes 423 fizeram parte do período pré-pandemia e 321 do período durante a pandemia, sendo estatisticamente diferente, conforme a Tabela 1 e Figura 1. Quanto ao turno de admissão no turno da manhã e tarde houve redução dos casos, mas sem diferença estatística, no entanto os casos admitidos no turno da noite tiveram redução significante no período da pandemia.

Em relação às variáveis demográficas o sexo masculino se apresentou, em relação ao feminino, com maior número de casos em ambos os períodos, entretanto houve diminuição estatisticamente significativa apenas para os indivíduos do sexo masculino entre os períodos. A idade teve aumento na média no período durante a pandemia, sendo estatisticamente diferente 
entre os dois períodos (Tabela 1 e Figura 2). Contudo, quando comparada a idade entre indivíduos do mesmo sexo, apenas o masculino demonstrou diferença estatística (Tabela 1 e Figura 3).

Tabela 1. Pacientes com trauma bucomaxilofacial, sexo, idade média e turno de admissão no período pré-pandemia e durante a pandemia.

\begin{tabular}{lccc}
\hline Período & Pré pandemia & Durante a pandemia & p-value \\
\hline Pacientes com Trauma bucomaxilofacial & $423(56,85)$ & $321(43,15)$ & $\mathbf{0 . 0 3} *$ \\
\hline Sexo & $303(71,63)$ & $220(68,54)$ & $\mathbf{0 . 0 0 5 *}$ \\
\hline Homem & $120(28,37)$ & $101(31,46)$ & 0.390 \\
\hline Mulher & $31.59(0-83)$ & $35.26(0-95)$ & $\mathbf{0 . 0 1 1} *$ \\
\hline Idade média (variação em anos) & & & 0.128 \\
\hline Turno de admissão & $97(22,93)$ & $72(22,43)$ & 0.327 \\
\hline Manhã & $145(34,28)$ & $123(38,32)$ & $\mathbf{0 . 0 3 7 *}$ \\
\hline Tarde & $181(42,79)$ & $126(39,25)$ & \\
\hline Noite & & & \\
\hline
\end{tabular}

Nota: *p>0,05. Fonte: Autores, com base nos dados da pesquisa.

A partir da Tabela 1 é visualizado a quantidade de pacientes atendidos pré-pandemia e durante a pandemia, bem como a diferença estatística entre os dois, que se mostrou diferente. Também é possível analisar a mudança na quantidade de homens e mulheres e que a redução de ocorrências do sexo masculino durante a pandemia mostrou-se estatisticamente menor. É observado o aumento na média de idade dos pacientes no período durante a pandemia, sendo estatisticamente diferente. Quanto ao turno de admissão os pacientes admitidos à noite tiveram redução significante.

Figura 1. Gráfico mostrando a variação de casos de trauma no período durante a pandemia e pré-pandemia.

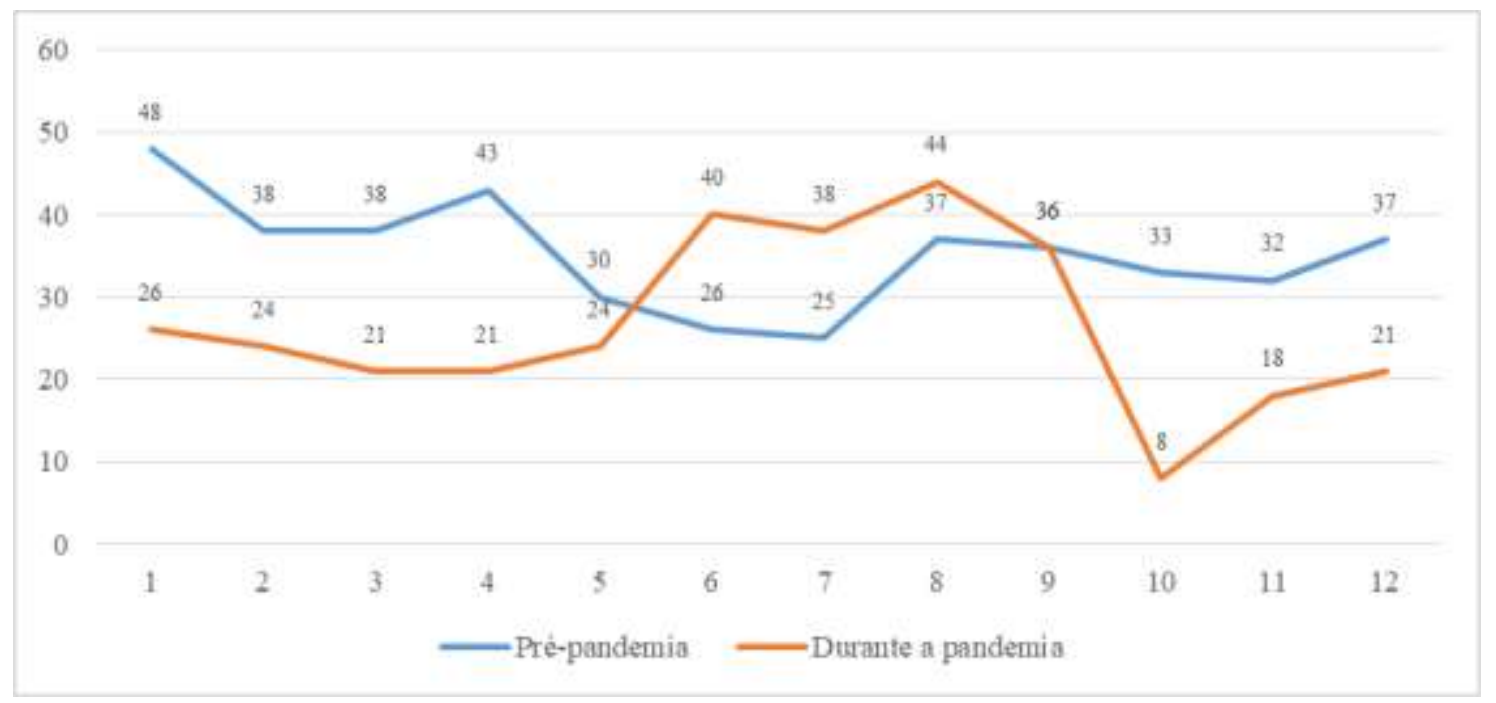

Fonte: Autores, com base nos dados da pesquisa.

Na Figura 1 é observado a variação quantitativa da ocorrência dos traumas e complicações avaliadas pelo serviço de cirurgia e traumatologia bucomaxilofacial do HUOP, ilustrando comparativamente a quantidade por mês do período prépandemia e durante a pandemia. 
Research, Society and Development, v. 10, n. 11, e236101119405, 2021

(CC BY 4.0) | ISSN 2525-3409 | DOI: http://dx.doi.org/10.33448/rsd-v10i11.19405

Figura 2. Gráfico mostrando a variação da média de idade.

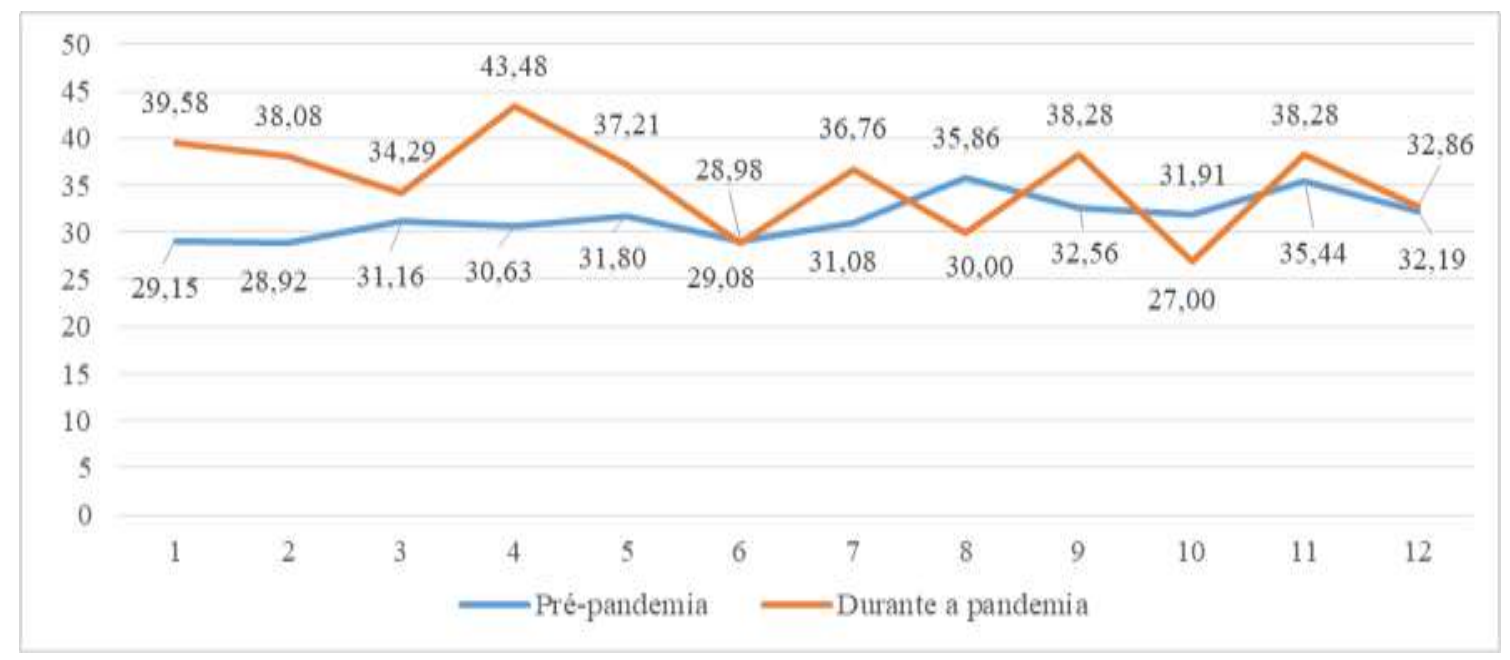

Fonte: Autores, com base nos dados da pesquisa.

A Figura 2 demonstra a variação da média de idade, por mês, em cada período analisado, das ocorrências avaliadas pelo serviço de cirurgia e traumatologia bucomaxilofacial do HUOP.

Figura 3. Gráfico demonstrando a variação do sexo no período pré-pandemia e durante a pandemia.

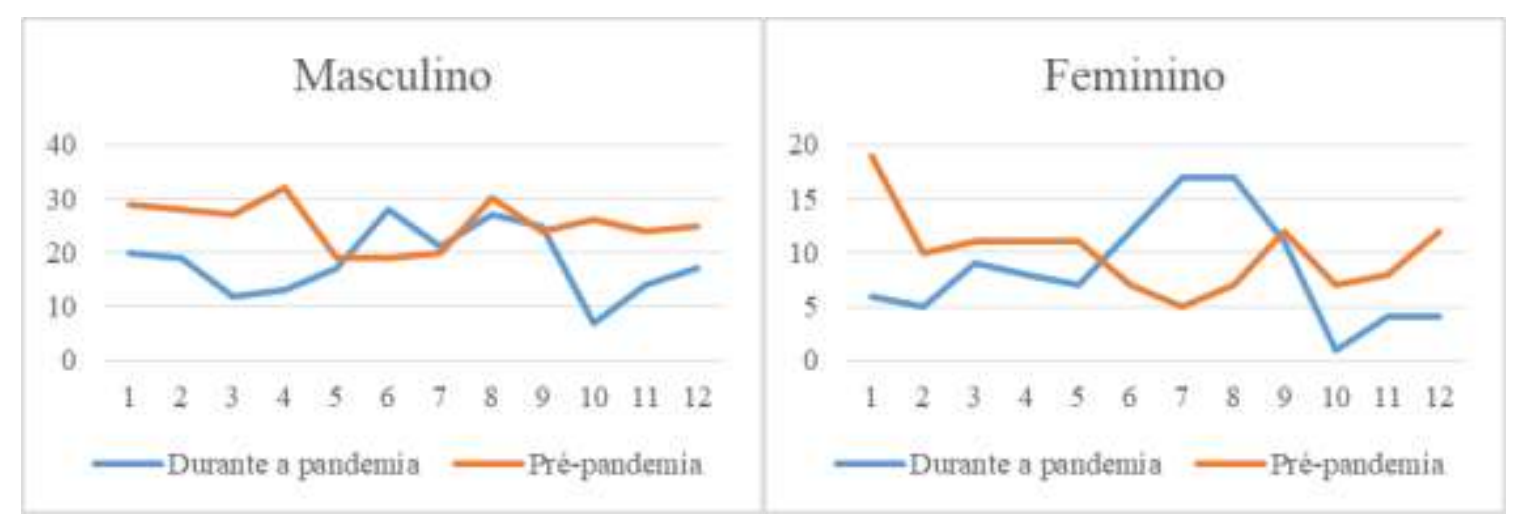

Fonte: Autores, com base nos dados da pesquisa.

Na Figura 3 pode-se observar a ocorrência dos traumas e complicações avaliadas pelo serviço de cirurgia e traumatologia bucomaxilofacial do HUOP, por quantidade em relação ao sexo, masculino ou feminino, e a quantidade por período em cada mês dos anos analisados.

Quanto à etiologia dos acidentes houve redução significativa apenas para os casos de agressão física e multifatorial (Tabela 2 e Figura 4). 
Tabela 2. Etiologia dos traumas e complicações avaliadas pelo serviço de cirurgia e traumatologia bucomaxilofacial do HUOP.

\begin{tabular}{llllll}
\hline Período & \multicolumn{2}{l}{ Pré pandemia } & \multicolumn{2}{c}{ Durante a pandemia } & p-value \\
\hline Etiologia & $\mathrm{N}$ & $\%$ & $\mathrm{~N}$ & $\%$ & 0.582 \\
\hline Acidente de trânsito & 119 & 28,13 & 109 & 33,96 & 0.299 \\
\hline Acidentes esportivos & 51 & 12,06 & 41 & 12,77 & 0.605 \\
\hline Quedas & 67 & 15,84 & 60 & 18,69 & 0.477 \\
\hline Acidente de Trabalho & 17 & 4,02 & 13 & 11,84 & $\mathbf{0 . 0 1 6 *}$ \\
\hline Agressão física & 64 & 15,13 & 38 & $\mathbf{1 3}$ & $\mathbf{0 . 0 1 1 *}$ \\
\hline Multifatorial & 97 & 22,93 & 49 & 11,43 & 0.447 \\
\hline Outros & 8 & 1,89 & 321 & & \\
\hline
\end{tabular}

Fonte: Autores, com base nos dados da pesquisa.

É possível analisar a etiologia dos traumas e complicações avaliadas pelo serviço de cirurgia e traumatologia bucomaxilofacial do HUOP no período pré-pandemia e durante a pandemia pela Tabela 2 a quantidade, bem como o valor em percentual e quanto é representado estatisticamente pelo valor de $\mathrm{p}$. 
Figura 4. Etiologia das ocorrências pré-pandemia e durante a pandemia.
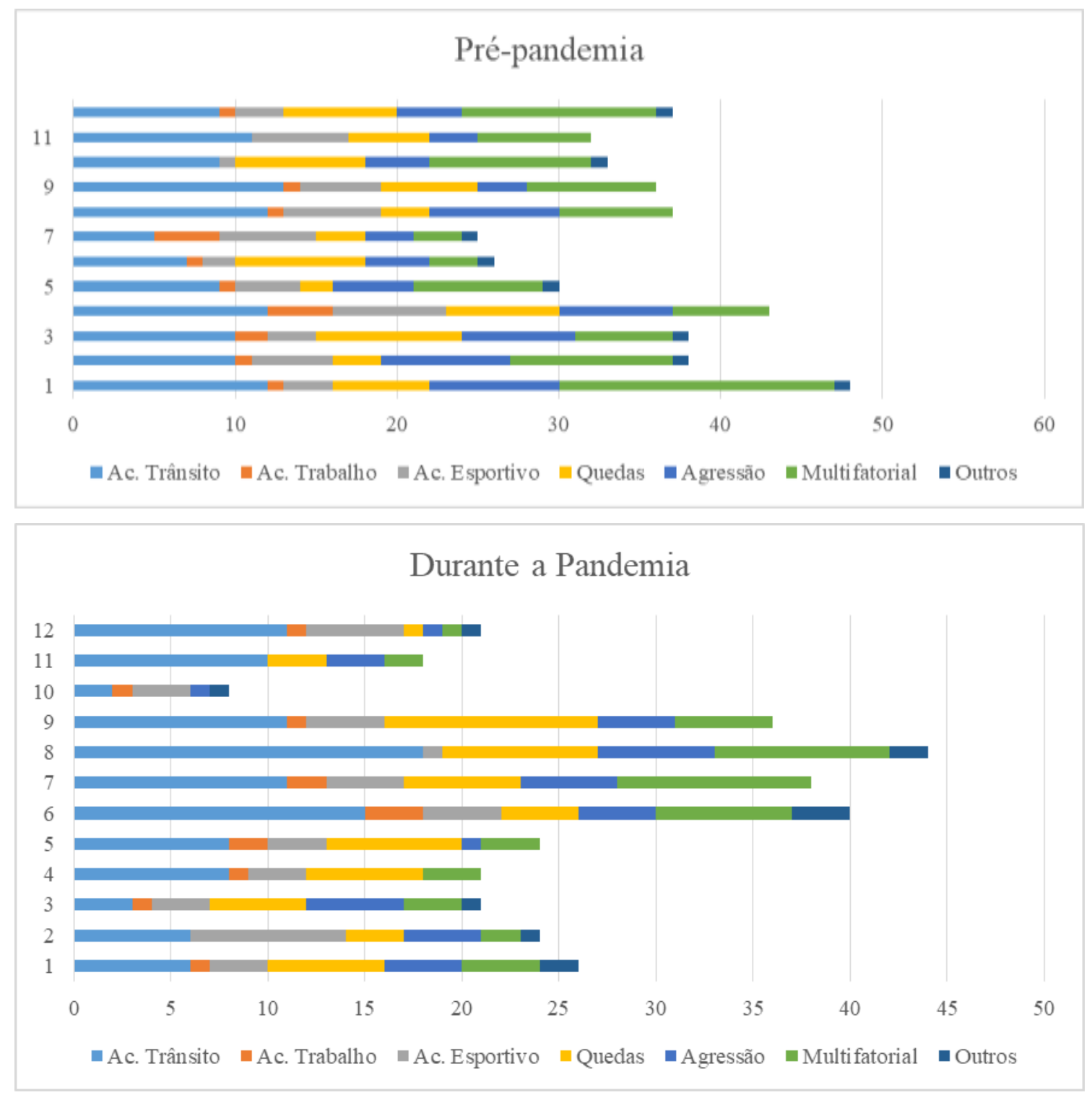

Fonte: Autores, com base nos dados da pesquisa.

A partir da Figura 4 é possível comparar visualmente a mudança na etiologia das ocorrências de trauma bucomaxilofacial de acordo com cada mês analisado e o número total de ocorrências.

\section{Discussão}

O Hospital Universitário do Oeste do Paraná (HUOP) é classificado como um hospital de médio porte e alta complexidade com atendimento de urgência e emergência, sendo referência para 25 municípios da região Oeste e Sudoeste do estado do Paraná, Brasil, prestando atendimento em diversas especialidades profissionais. Dentre elas, é referência para trauma e complicações na área bucomaxilofacial da região.

Houve diminuição das ocorrências relacionadas aos traumas e complicações bucomaxilofaciais durante a pandemia pela COVID-19, assim como ocorreu em outros centros de referência para o mesmo tipo de trauma (Press, 2021; Vishal et al., 2020; Yeung, Brandsma, Karst, Smith, \& Fan, 2021). Além disso as maiores reduções se relacionam com períodos de lockdown (Thiem et al., 2021). Esta diminuição também se relaciona com outras especialidades que tratam de trauma, como a 
ortopedia (Park, Sugand, Nathwani, Bhattacharya, \& Sarraf, 2020). Alguns centros de referência para trauma bucomaxilofacial trazem relatos de aumento no número de casos em 2020, mesmo com a pandemia os pacientes positivos para o vírus SARSCoV-2, sem sintomas ou com sintomas leves, não apresentaram interferência no seu tratamento (Canzi et al., 2020).

A literatura apresenta estudos, que também ocorreram no Brasil e descreveram a incidência de trauma, porém não em pandemia que mostraram os acidentes de trânsito como a principal etiologia dos traumas (Scannavino, Santos, Neto, \& Novo, 2013), assim como o encontrado no estudo, entretanto, outros mostram maior incidência nos casos de agressão (Zamboni, Wagner, Volkweis, Buchmann, \& Bavaresco, 2017) contrariando o que foi encontrado no presente estudo. Em nível mundial, outros locais apresentaram aumento para agressões, principalmente as de origem doméstica, durante o período de pandemia (Stanisce et al., 2021)

Quanto à etiologia, os casos de agressão diminuíram significativamente, assim como em estudos anteriores (Press, 2021). Além disso, quando analisado o horário com maior admissão de pacientes no hospital, o que teve maior redução foi o noturno. Estes fatos podem estar relacionados com a diminuição da circulação das pessoas em vias públicas instituídas pelo toque de recolher, bem como a não abertura de estabelecimentos como bares, impostos pelos decretos estaduais e municipais que visavam a redução dos casos de coronavírus. Essa redução também ocorreu em outros locais no mesmo período (Blackhall et al., 2020).

Ainda relacionado à etiologia os casos classificados como multifatoriais incluíam infecções odontogênicas e outros procedimentos eletivos de exodontias, enxertos ósseos e cirurgias ortognáticas. As cirurgias eletivas foram suspensas em diversos momentos do período em pandemia (Secretaria do Estado da Saúde do Paraná, 2020), o que provocou grande diminuição na admissão de pacientes com esta etiologia.

Os procedimentos bucomaxilofaciais estão relacionados com a propagação de aerossóis e, por isso, o serviço de urgência hospitalar deve estar focado em diagnóstico de doenças malignas, infecções profundas de cabeça e pescoço, redução de fraturas expostas, cominuídas e com prejuízo funcional e estético ao paciente, para que os recursos limitados sejam usados de forma eficaz (Zimmermann \& Nkenke, 2020). Alguns estudos trazem o uso de telemedicina nesta época de pandemia para auxiliar na triagem, antes do deslocamento do paciente, utilizando exames de imagem e informações referentes aos sinais e sintomas, contribuindo assim para o atendimento na unidade hospitalar apenas dos pacientes que realmente necessitam (Blackhall et al., 2020; Robiony et al., 2021).

\section{Conclusão}

O período de pandemia mostrou associação com a redução da ocorrência de pacientes com atendidos pelo serviço de cirurgia e traumatologia bucomaxilofacial do HUOP. A redução de casos admitidos no período noturno assim como de pacientes do sexo masculino pode ser atribuída como resultado das restrições de circulação de pessoas nas vias públicas e estabelecimentos que a pandemia impôs. O efeito do consequente distanciamento social, sugere uma diminuição no trauma bucomaxilofacial devido à agressão física.

Como possíveis trabalhos futuros, aponta-se a realização de um levantamento dos procedimentos cirúrgicos realizados pela especialidade de Cirurgia e traumatologia bucomaxilofacial no período pré-pandemia e durante a pandemia, além disso também poderá ser realizada uma nova comparação entre períodos após a exclusão das medidas restritivas contra a pandemia pela COVID-19.

\section{Referências}

Blackhall, K. K., Downie, I. P., Ramchandani, P., Kusanale, A., Walsh, S., Srinivasan, B., ... Singh, R. P. (2020). Provision of Emergency Maxillofacial Service During the COVID-19 Pandemic: A Collaborative Five Centre UK Study. British Journal of Oral and Maxillofacial Surgery, 58(6), 698-703. https://doi.org/10.1016/j.bjoms.2020.05.020 
Canzi, G., Ponti, E. De, Corradi, F., Bini, R., Novelli, G., Bozzetti, A., \& Sozzi, D. (2020). Epidemiology of Maxillo-Facial Trauma During COVID-19 Lockdown : Reports From the Hub Trauma Center in Milan. Craniomaxillofacial Trauma \& Reconstruction, 1-7. https://doi.org/10.1177/1943387520983119

Governo do Estado do Paraná. Decreto $n^{\circ} 4230$ de 16 de março de 2020. Dispõe sobre as medidas para enfrentamento da emergência de saúde pública de importância internacional decorrente do Coronavírus - COVID-19. , Pub. L. No. 4230 (2020). Brasil.

Lunardi, A. C. (2020). Manual de pesquisa clínica aplicada à saúde. Blucher.

Onder, G., Rezza, G., \& Brusaferro, S. (2020). Case-Fatality Rate and Characteristics of Patients Dying in Relation to COVID-19 in Italy. JAMA - Journal of the American Medical Association, 323(18), 1775-1776. https://doi.org/10.1001/jama.2020.4683

Park, C., Sugand, K., Nathwani, D., Bhattacharya, R., \& Sarraf, K. M. (2020). Impact of the COVID-19 pandemic on orthopedic trauma workload in a London level 1 trauma center: the "golden month": The COVid Emergency Related Trauma and orthopaedics (COVERT) Collaborative. Acta Orthopaedica, 91(5), 556-561. https://doi.org/10.1080/17453674.2020.1783621

Press, S. G. (2021). What is the Impact of the 2020 Coronavirus Lockdown on Maxillofacial Trauma? Journal of Oral and Maxillofacial Surgery, 1-5. https://doi.org/10.1016/j.joms.2021.01.010

Robiony, M., Bocin, E., Sembronio, S., Costa, F., Arboit, L., \& Tel, A. (2021). Working in the era of COVID-19: An organization model for maxillofacial surgery based on telemedicine and video consultation. Journal of Cranio-Maxillofacial Surgery, 49(4), 323-328. https://doi.org/10.1016/j.jcms.2021.01.027

Rothe, C., Schunk, M., Sothmann, P., Bretzel, G., Froeschl, G., Wallrauch, C., ... Hoelscher, M. (2020). Transmission of 2019-nCoV Infection from an Asymptomatic Contact in Germany. New England Journal of Medicine, 382(10), 970-971. https://doi.org/10.1056/NEJMc2001468

Scannavino, L., Santos, F., Neto, J., \& Novo, L. (2013). Análise epidemiológica dos traumas bucomaxilofaciais de um serviço de emergência. Rev. Cir. Traumatol. Buco-Maxilo-Fac., 13(4), 95-100.

Secretaria do Estado da Saúde do Paraná. RESOLUÇÃO SESA No 338/2020. Regulamenta o disposto nos arts. $1^{\circ}, 2^{\circ}, 3^{\circ}, 10,13$ e 15 do Decreto Estadual n ${ }^{\circ}$ 4.230, 16 de março de 2020, para implementar medidas de enfrentamento da emergência em saúde pública de importância nacional e internacional decorr. , (2020). Brasil.

Stanisce, L., Fisher, A. H., Choi, B. Y., Newman, A., Wang, J. L., \& Koshkareva, Y. (2021). How Did the COVID-19 Pandemic Affect Trends in Facial Trauma ? Craniomaxillofacial Trauma \& Reconstruction, 1-7. https://doi.org/10.1177/19433875211022574

Thiem, Polsak, D. G. E., Römer, Gielisch, P., Blatt, Al-Nawas, \& Kämmerer. (2021). The impact of the COVID - 19 pandemic on the dental - maxillofacial emergency service of a German university hospital in the year 2020. Clinical Oral Investigations, (0123456789). https://doi.org/10.1007/s00784-021-04010-7

Vishal, Prakash, O., Rohit, Prajapati, V. K., Shahi, A. K., \& Khaitan, T. (2020). Incidence of Maxillofacial Trauma Amid COVID-19: A Comparative Study. Journal of Maxillofacial and Oral Surgery. https://doi.org/10.1007/s12663-020-01484-y

World Health Organization. (2020a). Infection prevention and control during health care when COVID-19 is suspected: interim guidance.

World Health Organization. (2020b). Rational use of personal protective equipment (PPE) for coronavirus disease (COVID-19): interim guidance.

Wu, Z., Zhao, S., Yu, B., Chen, Y., Wang, W., Song, Z., ... Zhang, Y. (2020). A new coronavirus associated with human respiratory disease in China. Nature, 579(March), 265-269. https://doi.org/10.1038/s41586-020-2008-3

Yang, Y., Soh, H. Y., Cai, Z. G., Peng, X., Zhang, Y., \& Guo, C. Bin. (2020). Experience of Diagnosing and Managing Patients in Oral Maxillofacial Surgery during the Prevention and Control Period of the New Coronavirus Pneumonia. Chinese Journal of Dental Research, 23(1), 57-62. https://doi.org/10.3290/j.cjdr.a44339

Yeung, E., Brandsma, D. S., Karst, F. W., Smith, C., \& Fan, K. F. M. (2021). The influence of 2020 coronavirus lockdown on presentation of oral and maxillofacial trauma to a central London hospital. British Journal of Oral and Maxillofacial Surgery, 59(1), 102-105. https://doi.org/10.1016/j.bjoms.2020.08.065

Zamboni, R. A., Wagner, J. C. B., Volkweis, M. R., Buchmann, E. M., \& Bavaresco, C. S. (2017). Epidemiological study of facial fractures at the Oral and Maxillofacial Surgery Service, Santa Casa de Misericordia Hospital Complex, Porto Alegre - RS - Brazil. Revista Do Colégio Brasileiro de Cirurgia, 44(5), 491-497. https://doi.org/10.1590/0100-69912017005011

Zimmermann, M., \& Nkenke, E. (2020). Approaches to the management of patients in oral and maxillofacial surgery during COVID-19 pandemic. Journal of Cranio-Maxillofacial Surgery, 48(5), 521-526. https://doi.org/10.1016/j.jcms.2020.03.011 